\title{
New technique for system simplification using Cuckoo search and ESA
}

\author{
AFZAL SIKANDER $^{1,2, * \mathbb{D}}$ and RAJENDRA PRASAD ${ }^{1}$ \\ ${ }^{1}$ Department of Electrical Engineering, Indian Institute of Technology Roorkee, Roorkee 247667, India \\ ${ }^{2}$ Department of Electrical Engineering, Graphic Era University, Dehradun 248002, India \\ e-mail: afs26dee@iitr.ac.in; afzal.sikander@hotmail.com
}

MS received 8 December 2015; revised 21 September 2016; accepted 28 January 2017; published 17 August 2017

\begin{abstract}
In this study, a new technique is suggested for simplification of linear time-invariant systems. Motivated by optimization and various system simplification techniques available in the literature, the proposed technique is formulated using Cuckoo search in combination with Lévy flight and Eigen spectrum analysis. The efficacy and powerfulness of the new technique is illustrated by three benchmark systems considered from previously published work and the results are compared in terms of performance indices.
\end{abstract}

Keywords. Cuckoo search; Eigen spectrum analysis; optimization; reduced order modelling.

\section{Introduction}

Often, high-order differential equations or difference equations are used to represent the mathematical model of a system. By using these differential/difference equations it is difficult to analyse the system. Hence, for simplification of such systems, the order of these equations must be reduced such that the simplified/lower order system retains all necessary properties of the higher order system. Therefore, for better understanding of the system, this lower order system is used to analyse behaviour of higher order system by which simulation time reduces and design work become easier. Hence, modal order reduction is the most widely used concept in different fields of engineering due to its simplicity in control designing, low cost, computational speed, satisfactory performance and because it is easy to understand in many applications. These interesting features have made the modal order reduction as an attractive research topic in the past several decades. A detailed survey on this topic is given by Sinha and Lastman [1].

Recently, Ghosh and Senroy [2] proposed a technique for system simplification that is based on balanced truncation technique. The advantage of factor division algorithm and stability equation method is being utilized for reduced order modelling by Sikander and Prasad [3]. Also, Desai and Prasad [4] proposed a new technique for linear time-invariant (LTI) systems, which is based on Routh Approximation (RA) [5] and Big Bang-Big Crunch (BB-BC) algorithm [6] to obtain the lower order system. RA is used to evaluate the denominator polynomial of the lower order system to preserve the stability, whereas the numerator polynomial is evaluated using the BB-BC algorithm. Another composite technique was suggested by Parmar

*For correspondence et al [7], by which they demonstrated the advantages of combining Eigen spectrum analysis (ESA) and factor division algorithm. This approach is applied on the system having real poles only and extended for MIMO systems also. ESA along with Padé approximation (ESA \& PA) [8] and Cauer second from (ESA \& CAUER-II) [9] is proposed by the authors for system simplification. The major drawback of these techniques is that in some cases, the lower order model becomes a non-minimum phase model.

Also, Parmar et al [10] suggested a new technique to obtain a stable lower order model, in which the coefficients of denominator polynomial are evaluated by stability equation technique and the numerator polynomial is evaluated by a genetic algorithm. They emphasized on preserving the stability. One more composite technique was suggested by Vishwakarma and Prasad [11], where denominator of the lower order system is evaluated by clustering technique and its numerator is obtained by Padé approximation technique. This technique also produces a non-minimum phase lower order system. Recently, stability equation technique along with particle swarm optimization was suggested by Sikander and Prasad [12]. They used the concept of integral square error (ISE) minimization. Despite having so many order reduction techniques, no technique provides satisfactory results so far for all systems. Hence, there is a scope for finding efficient new algorithms for system simplification.

Therefore, this paper contributes a new technique based on ESA and Cuckoo search (ESA \& CS) for simplification of linear dynamical systems. The coefficients of the denominator polynomial of the lower order system are calculated by ESA whereas the coefficients of the numerator polynomial are obtained by minimizing the ISE using a new meta-heuristic search algorithm called Cuckoo search. In this technique, stability of the system is retained and it is computationally very 
simple. Comparative analysis of the results obtained using the proposed technique is presented. To analyse the performance of the lower order systems, three performance indices, ISE, integral absolute error (IAE) and integral of time multiplied by absolute error $(I T A E)$, are used in this study.

\section{Problem statement}

Let us consider an $n$th order single input single output system represented as follows:

$$
G_{n}(s)=\frac{N_{n-1}(s)}{D_{n}(s)}=\frac{\sum_{i=0}^{n-1} f_{i} s^{i}}{\sum_{i=0}^{n} h_{i} s^{i}}
$$

where $f_{i}$ and $h_{i}$ are constants and let us assume $f_{0}=h_{0}$ for unity steady-state output response when the system is subjected to unit step input. The objective is to evaluate the unknown scalar constants of lower order system $(k$ th) $(k<n)$ described by the following equation:

$$
R_{k}(s)=\frac{N_{k-1}(s)}{D_{k}(s)}=\frac{\sum_{i=0}^{k-1} c_{i} s^{i}}{\sum_{i=0}^{k} d_{i} s^{i}}
$$

where $c_{i}$ and $d_{i}$ are unknown constants.

\section{Proposed methodology}

The proposed algorithm for system simplification or to obtain reduced order model is divided into the following two steps.
Step 1: Calculation of denominator polynomial of lower order system using ESA [7].

Step 1.1: Considering denominator polynomial of the original system and rearranging in the following form:

$$
D_{n}(s)=\left(s+p_{1}\right)\left(s+p_{2}\right) \ldots\left(s+p_{n}\right)
$$

such that $-p_{1}<-p_{2}<\cdots<-p_{n}$ are the poles of the higher order original system $G_{n}(s)$.

Step 1.2: Represent the poles (Eigen Spectrum Points (ESP)) of $G_{n}(s)$ in Eq. (3) on the negative real axis of the $s$ plane as shown in figure 1.

Step 1.3: Calculate the pole centroid given by

$$
\begin{gathered}
\sigma_{p}=\frac{\sum \text { absolute value of real part of poles }}{\text { number of poles }} \\
\sigma_{p}=\frac{\sum_{j=1}^{n}\left|\operatorname{Re} p_{j}\right|}{n}
\end{gathered}
$$

Step 1.4: Determine the system stiffness $K$ using the following formula:

$$
K=\frac{\left|\operatorname{Re} p_{1}\right|}{\left|\operatorname{Re} p_{n}\right|} \quad\left|\operatorname{Re} p_{1}\right|<\left|\operatorname{Re} p_{2}\right|<\cdots<\left|\operatorname{Re} p_{n}\right| .
$$

Step 1.5: Computation of $\operatorname{Re} p_{k}{ }^{\prime}, M$.

Let $\sigma_{p}{ }^{\prime}, K^{\prime}$ be the pole centroid and system stiffness of $R_{k}(s)$, respectively. If $\sigma_{p}^{\prime}=\sigma_{p}$ and $K^{\prime}=K$ then

$$
K=\frac{\left|\operatorname{Re} p_{1}^{\prime}\right|}{\left|\operatorname{Re} p_{k}^{\prime}\right|}=K^{\prime} \text { and } \sigma_{p}=\frac{\sum_{j=1}^{k}\left|\operatorname{Re} p_{j}^{\prime}\right|}{k}=\sigma_{p}^{\prime}
$$

$\operatorname{Re} p_{j}^{\prime}$ are the real parts of poles of $R_{k}(s) ; \sigma_{p}$ can be rewritten as follows:

$$
\begin{aligned}
& \sigma_{p}=\frac{\operatorname{Re} p_{1}^{\prime}+\operatorname{Re}_{2}^{\prime}+\operatorname{Re}_{3}^{\prime}+\cdots+\operatorname{Re}_{k-1}^{\prime}+\operatorname{Re}_{k}^{\prime}}{k} \\
& =\frac{\operatorname{Re} p_{1}^{\prime}+\left(\operatorname{Re} p_{1}^{\prime}+M\right)+\left(\operatorname{Re} p_{1}^{\prime}+2 M\right)+\cdots+\left(\operatorname{Re} p_{1}^{\prime}+(k-2) M\right)+\operatorname{Re} p_{k}^{\prime}}{k} \\
& =\operatorname{Re} p_{1}^{\prime}+\operatorname{Re}_{1}^{\prime}(k-2)+\left(M+2 M+\cdots+(k-2) M+\operatorname{Re} p_{k}^{\prime}\right) \\
M & =\frac{\operatorname{Re} p_{k}^{\prime}-\operatorname{Re} p_{1}^{\prime}}{(k-1)}
\end{aligned}
$$

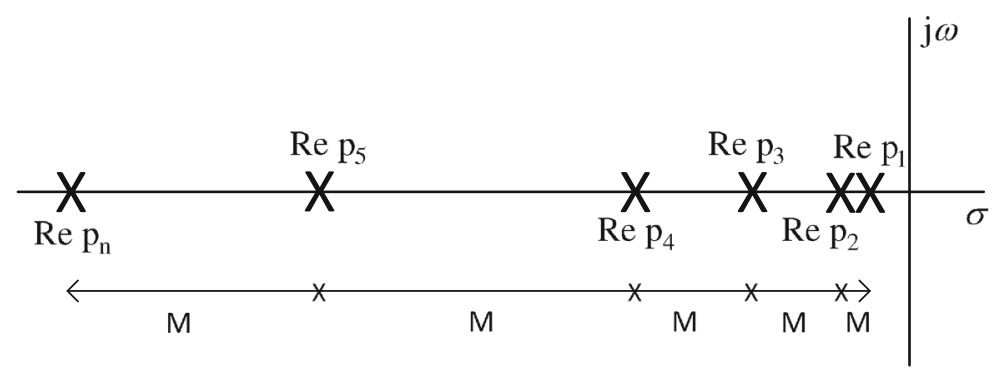

Figure 1. Representation of ESP of the original system. 
Replacing $\operatorname{Re} p_{1}^{\prime}=K \operatorname{Re} p_{k}^{\prime}$

$$
M(k-1)=\operatorname{Re} p_{k}^{\prime}-K \operatorname{Re} p_{k}^{\prime} .
$$

We have

$$
\begin{gathered}
N=\sigma_{p} k \\
Q M=(M+2 M+\cdots+(k-2) M) .
\end{gathered}
$$

Substituting the values of $\sigma_{p}$, and $Q M$, Eq. (7) gives

$$
N=K \operatorname{Re}_{k}^{\prime}(k-1)+\operatorname{Re} p_{k}^{\prime}+Q M .
$$

Equations (6) and (8) can be rearranged in the following matrix form:

$$
\left[\begin{array}{lr}
K(k-1)+1 & Q \\
(1-K) & (1-k)
\end{array}\right]\left[\begin{array}{c}
\operatorname{Re} p_{k}^{\prime} \\
M
\end{array}\right]=\left[\begin{array}{c}
N \\
0
\end{array}\right]
$$

The value of $\operatorname{Re}_{k}^{\prime}, M$ is obtained by solving Eq. (9).

Step 1.6: Calculate ESP from $\operatorname{Re} p_{k}^{\prime}$, and $M$, and form denominator polynomial $D_{k}(s)$ of lower order system.

Step 2: Calculation of numerator polynomial of lower order system using Cuckoo search algorithm [13].

Step 2.1: Specify the fitness function as given in Eq. (12) and the number of chosen variables (say $q$ ) as per Eq. (2) along with their range. Set the probability of the worst nests and step size also. After initialization of a population of $p$ host nests the problem is summarized as follows:

minimize fitness function, i.e., ISE $Z_{\alpha}$, subject to $c_{i L}<c_{i}<c_{i U}$ and $d_{i L}<d_{i}<d_{i U}$ where $c_{i L}, d_{i L}$ and $c_{i U}, d_{i U}$ are the lowest and highest values of the chosen variables, respectively, and $i=0,1, \ldots, q$.

Step 2.2: Obtain the value of $Z_{\alpha}$ for a randomly selected cuckoo $(\alpha)$ and select a nest $(\beta)$ randomly among $p$.

Step 2.3: If $\left(Z_{\alpha}>Z_{\beta}\right)$ then interchange $\beta$ by the current obtained solution, else go to the next step.

Step 2.4: Check whether the predefined stopping criterion has been reached or the maximum generation has occurred or not. If yes, then the solution obtained in the current generation would be the best solution; otherwise, go to the next step.

Step 2.5: Abandon a fraction of worst nests with optimal value of probability $p_{a}$ and step size $a$.

Step 2.6: Using Eq. (10), the obtained solution must be updated by calculating $Y_{i}^{(t+1)}$ and repeating this algorithm, until the predefined condition or the maximum generation is obtained.

For simplicity, the fraction of $p_{a}$ of $n$ nests, which are interchanged new nests, is considered as an approximation of the previous assumption.

The Lévy flight can be represented by the following relation for the generation of new solution $Y(t+1)$ of cuckoo $i[14]$ :

$$
Y_{i}^{(t+1)}=Y_{i}^{(t)}+a \otimes \operatorname{Lévy}(\lambda)
$$

where $a(a>0)$ is a step size that is related to the level of the problem optimized by the technique. The random step size of the Lévy flights is calculated as follows:

$$
\text { Lévy } \tilde{u}=t^{-\lambda} \quad(1<\lambda \leq 3) .
$$

Furthermore, the efficacy of the lower order system obtained by the proposed technique is evaluated using the following performance indices:

$$
\begin{gathered}
I S E=\int_{0}^{\infty}\left[g_{n}(t)-r_{k}(t)\right]^{2} d t \\
\text { IAE }=\int_{0}^{\infty}\left|g_{n}(t)-r_{k}(t)\right| d t \\
\text { ITAE }=\int_{0}^{\infty} t \cdot\left|g_{n}(t)-r_{k}(t)\right| d t
\end{gathered}
$$

where ISE, IAE and ITAE are integral square error, integral of absolute error and integral of time multiplied by absolute error, respectively; $g_{n}(t)$ and $r_{k}(t)$ are step responses of higher and lower order system, respectively.

\section{Numerical examples and results}

Example 1 Let us consider a 10th order model [8] for reduction that is as follows:

$$
G_{10}(s)=\frac{540.70748 \times 10^{17}}{\prod_{i=1}^{10}\left(s+p_{i}\right)}
$$

where

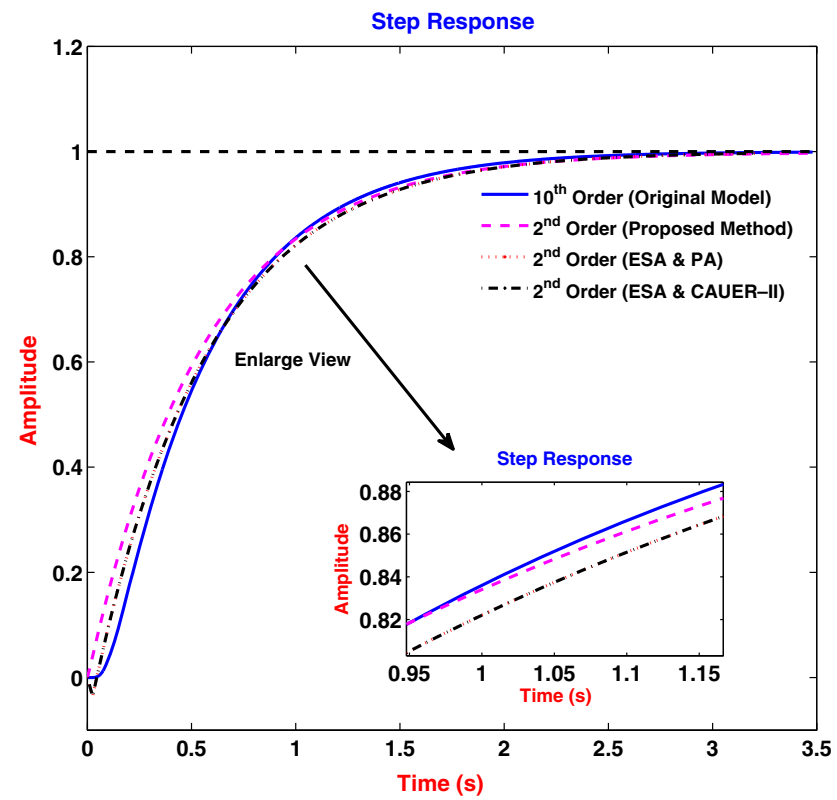

Figure 2. Comparison of time response of higher and lower order systems with different reduction techniques for Example 1. 
Table 1. Comparison of different reduction techniques for Example 1.

\begin{tabular}{|c|c|c|c|c|c|c|}
\hline Reduction technique & $t_{r}(\mathrm{~s})$ & $t_{s}(\mathrm{~s})$ & $M_{p}(\%)$ & $I S E$ & $I A E$ & ITAE \\
\hline Original system & 1.0901 & 2.0314 & 0 & - & - & - \\
\hline Proposed & 1.2152 & 2.1665 & 0 & $5.10 \times 10^{-4}$ & $7.45 \times 10^{-3}$ & $58.92 \times 10^{-3}$ \\
\hline ESA \& PA [8] & 1.2153 & 2.1743 & 0 & 1.3646 & $4.56 \times 10^{-2}$ & $36.11 \times 10^{-2}$ \\
\hline ESA \& CAUER-II [9] & 1.2253 & 2.1943 & 0 & $1.5 \times 10^{-3}$ & $4.56 \times 10^{-2}$ & $36.11 \times 10^{-2}$ \\
\hline
\end{tabular}

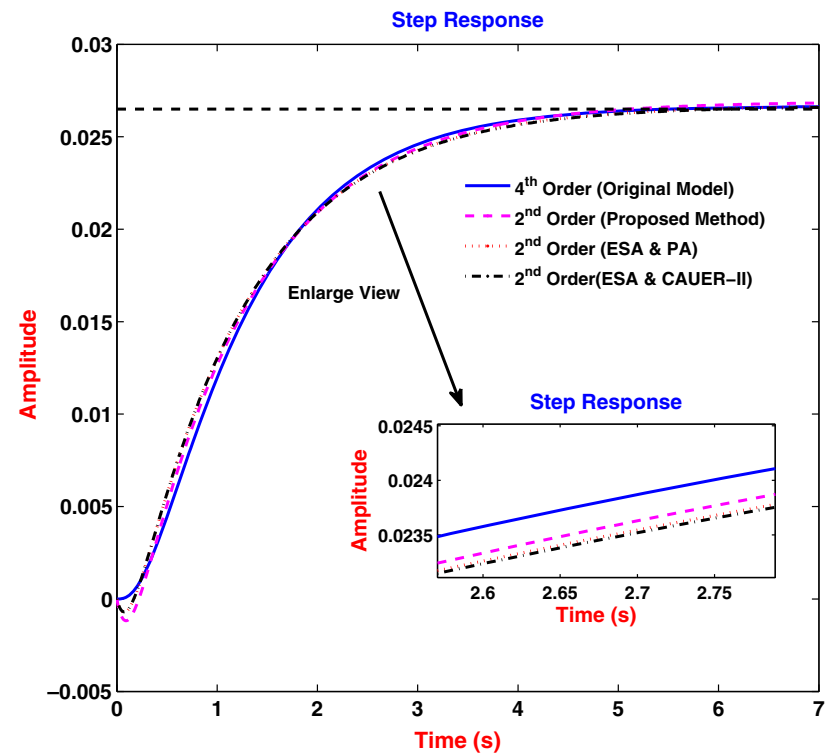

Figure 3. Comparison of time response of higher and lower order systems with different reduction techniques for Example 2.

$p_{1}=2.04, p_{2}=18.3, p_{3}=50.13, p_{4}=95.15, \quad p_{5}=$ $148.85, p_{6}=205.16, p_{7}=257.21, p_{8}=298.03, p_{9}=$ $320.97, p_{10}=404.16$.

On applying the proposed technique, the following lower second order model is obtained with $I S E=5.10 \times 10^{-4}$ :

$$
R_{2}(s)=\frac{0.1729 s+646.7}{s^{2}+360 s+647.6}
$$

whereas the lower second order system obtained by (ESA \& PA) [8] with $I S E=1.3646$ is represented as follows:

$$
R_{2}(s)=\frac{-28.367 s+647.60193}{s^{2}+359.999 s+647.60193}
$$

The step responses of higher order system and lower order system obtained by proposed technique (ESA \& CS), (ESA \& PA) [8] and (ESA \& CAUER-II) [9] are shown in figure 2. It is clearly observed that the lower order system obtained by the proposed technique provides good approximation to the higher order system. Also, a comparison of lower order system obtained by the proposed technique and by alternative techniques in terms of rise time $\left(t_{r}\right)$, settling time $\left(t_{s}\right)$, maximum peak overshoot $\left(M_{p}\right)$ and performance indices is presented in table 1 for Example 1. It is noticed that the proposed technique provides lesser ISE value, i.e., $5.10 \times 10^{-4}$, as compared with the values obtained using other techniques and in recently published work available in the literature, i.e., 1.3646 by (ESA \& PA) [8] and $1.5 \times 10^{-3}$ by (ESA \& CAUER-II) [8]. Therefore, it is clearly noticed that the proposed technique is much better as compared with other techniques available in the literature.

Example 2 The second model [8], which is to be reduced by the proposed technique, is given as follows:

$$
G_{4}(s)=\frac{s+4}{s^{4}+19 s^{3}+113 s^{2}+245 s+150}
$$

On applying the proposed technique, the lower second order system with $I S E=8.176 \times 10^{-7}$ is obtained as follows:

$$
R_{2}(s)=\frac{-0.03 s+0.2007}{s^{2}+9.5 s+7.459}
$$

whereas the lower second order system with ISE = $2.7663 \times 10^{-5}$ obtained by (ESA \& PA) [8] is represented

\begin{tabular}{|c|c|c|c|c|c|c|}
\hline Reduction technique & $t_{r}(\mathrm{~s})$ & $t_{s}(\mathrm{~s})$ & $M_{p}(\%)$ & $I S E$ & $I A E$ & $I T A E$ \\
\hline Original system & 2.3697 & 4.3584 & 0 & - & - & - \\
\hline Proposed & 2.5473 & 4.7127 & 0 & $8.176 \times 10^{-7}$ & $2.5 \times 10^{-3}$ & $4.24 \times 10^{-2}$ \\
\hline ESA \& PA [8] & 2.5597 & 4.7261 & 0 & $2.766 \times 10^{-5}$ & $4.10 \times 10^{-3}$ & $7.03 \times 10^{-2}$ \\
\hline ESA \& CAUER-II [9] & 2.5897 & 4.8961 & 0 & $1.6 \times 10^{-6}$ & $4.12 \times 10^{-3}$ & $7.08 \times 10^{-2}$ \\
\hline
\end{tabular}
as follows:

$$
R_{2}(s)=\frac{-0.02187 s+0.19915}{s^{2}+9.5 s+7.45868}
$$

Table 2. Comparison of different reduction techniques for Example 2. 
To demonstrate the efficacy of the proposed technique, the step responses of higher order system and lower order system obtained using the proposed technique (ESA \& CS), (ESA \& PA) [8] and (ESA \& CAUER-II) [9] are plotted in figure 3. It is perceived that the response of lower order system evaluated by the proposed technique is much closer
\& CAUER-II) [9]. The values of IAE and ITAE for the proposed technique are also found to be much lesser as compared with other techniques. Hence, the proposed technique exhibits better performance comparatively.

Example 3 Let us reduce an 8th order model [8], which is given as follows:

$$
G_{8}(s)=\frac{18 s^{7}+514 s^{6}+5982 s^{5}+36380 s^{4}+122664 s^{3}+222088 s^{2}+185760 s+40320}{s^{8}+36 s^{7}+546 s^{6}+4536 s^{5}+22449 s^{4}+67284 s^{3}+118124 s^{2}+109584 s+40320}
$$

than the responses of reduced models obtained by alternative techniques. In addition, the values of performance indices, such as ISE, IAE and ITAE values, along with rise time $\left(t_{r}\right)$, settling time $\left(t_{s}\right)$ and maximum peak overshoot $\left(M_{p}\right)$ of reduced models are also tabulated in table 2 for Example 2. Using the proposed technique, the ISE value for Example 2 is found to be $8.176 \times 10^{-7}$ whereas the lowest value of ISE, using the other well-known techniques, is only $2.7663 \times 10^{-5}(\mathrm{ESA} \& \mathrm{PA})[8]$ and $1.6 \times 10^{-6}(\mathrm{ESA}$

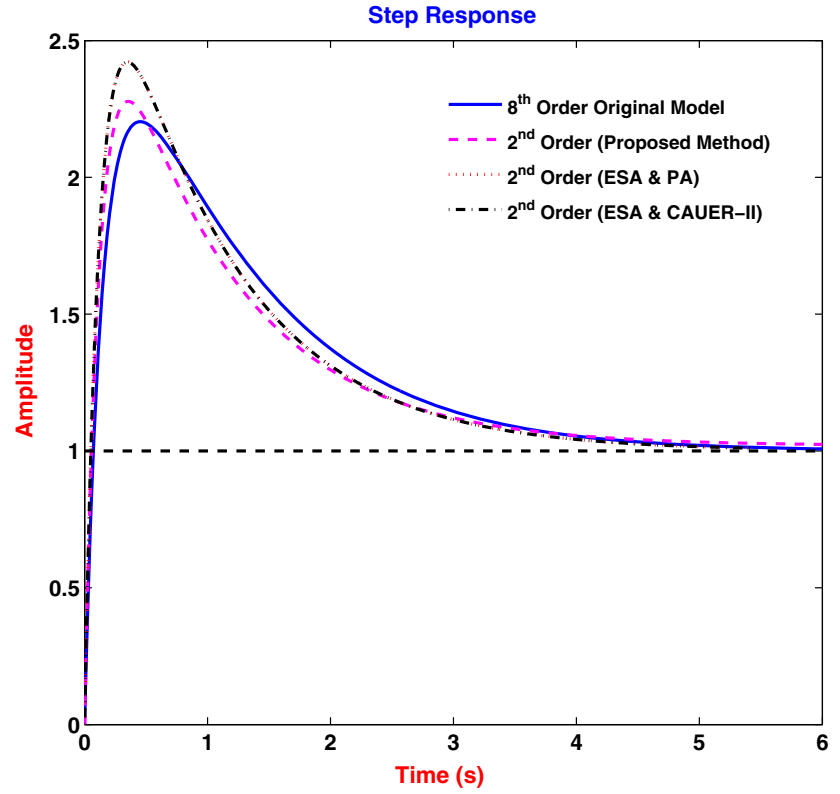

Figure 4. Comparison of time response of higher and lower order systems with different reduction techniques for Example 3.
On applying the proposed technique, the lower second order system obtained is

$$
R_{2}(s)=\frac{22.51 s+8.151}{s^{2}+9 s+8} .
$$

The same example is reduced by (ESA \& PA) [8] to obtain

$$
R_{2}(s)=\frac{24.1144 s+8}{s^{2}+9 s+8}
$$

The time responses of higher and lower order system when subjected to unit step input are depicted in figure 4 for Example 3. The enlarged view of the responses depicts the closeness of higher and lower order systems more clearly. To exhibit the excellence and effectiveness of the proposed technique, ISE, IAE and ITAE values along with rise time $\left(t_{r}\right)$, settling time $\left(t_{s}\right)$ and maximum peak overshoot $\left(M_{p}\right)$ of different lower order systems are also compared as depicted in table 3 . It is found that the proposed technique performs well comparatively.

\section{Conclusion}

In this study, a new simplification technique for LTI systems is presented, which is based on ESA and recently developed Cuckoo search algorithm. The coefficients of denominator polynomial of lower order system are calculated by ESA and the coefficients of numerator polynomial of lower order system are obtained by minimizing the ISE

\begin{tabular}{|c|c|c|c|c|c|c|}
\hline Reduction technique & $t_{r}(\mathrm{~s})$ & $t_{s}(\mathrm{~s})$ & $M_{p}(\%)$ & $I S E$ & $I A E$ & ITAE \\
\hline Original system & 0.0569 & 4.8201 & 120.35 & - & - & - \\
\hline Proposed & 0.0457 & 4.4002 & 123.56 & $3.63 \times 10^{-2}$ & $54.98 \times 10^{-3}$ & $10.71 \times 10^{-3}$ \\
\hline ESA \& PA [8] & 0.0409 & 4.3942 & 142.14 & 1.7924 & $30.07 \times 10^{-2}$ & 5.8571 \\
\hline ESA \& CAUER-II [9] & 0.0410 & 4.3945 & 142.15 & $4.81 \times 10^{-2}$ & $30.07 \times 10^{-2}$ & 5.8571 \\
\hline
\end{tabular}
between higher and lower order systems using Cuckoo

Table 3. Comparison of different reduction techniques for Example 3. 
search algorithm. The comparison of step response shows that the response of lower order system obtained by proposed technique is much closer to the response of higher order system as compared with other techniques. The efficacy and effectiveness of the proposed technique are also observed by comparing ISE, IAE and ITAE values of different lower order systems. Therefore, it is concluded that the proposed technique performs well as compared with other popular techniques available in the literature.

\section{References}

[1] Sinha N K and Lastman G J 1990 Reduced order models for complex systems-a critical survey. IETE Techn. Rev. 7(1): $33-40$

[2] Ghosh S and Senroy N 2013 Balanced truncation approach to power system model order reduction. Electr. Power Compon. Syst. 41: 747-764

[3] Sikander A and Prasad R 2015 Linear time-invariant system reduction using a mixed methods approach. Appl. Math. Model. 39(16): 4848-4858

[4] Desai S R and Prasad R 2013 A novel order diminution of LTI systems using Big Bang Big Crunch optimization and Routh Approximation. Appl. Math. Model. 37(16-17): 8016-8028
[5] Hutton M F and Friedland B 1975 Routh approximations for reducing order of linear, time-invariant systems. IEEE Trans. Autom. Control 20: 329-337

[6] Erol O K and Eksin I 2006 A new optimization method: Big Bang-Big Crunch. Adv. Eng. Softw. 37: 106-111

[7] Parmar G, Mukherjee S and Prasad R 2007 System reduction using factor division algorithm and eigen spectrum analysis. App. Math. Model. 31(11): 2542-2552

[8] Parmar G, Mukherjee S and Prasad R 2007 System reduction using eigen spectrum analysis and Padé approximation technique. Int. J. Comput. Math. 84(12): 1871-1880

[9] Parmar G, Mukherjee S and Prasad R 2007 A mixed method for large scale systems modelling using eigen spectrum analysis and Cauer second form. IETE J. Res. 53(2): 93-102

[10] Parmar G, Prasad R and Mukherjee S 2007 Order reduction of linear dynamic systems using stability equation method and GA. Int. J. Comput. Inf. Eng. 1(1): 26-32

[11] Vishwakarma C B and Prasad R 2008 System reduction using modified pole clustering and Padé approximation. In: Proceeding of the XXXII National Systems Conference, NSC, pp. 592-596

[12] Sikander A and Prasad R 2015 Soft computing approach for model order reduction of linear time invariant systems. Circuits Syst. Signal Process. 34(11): 3471-3487

[13] Yang X S and Deb S 2008 Nature-inspired metaheuristic algorithms. Luniver Press

[14] Yang X S and Deb S 2009 Engineering optimisation by cuckoo search. Int. J. Math. Model. Num. Optim. 1: 330-343 